\title{
Editorial
}

\section{Vacina Brasil e estratégias de formação e desenvolvimento em imunizações}

doi: 10.5123/S1679-49742019000200024

\section{Vacina Brasil Movement and immunization training and development strategies}

\author{
Vacina Brasil y estrategias de formación y desarrollo de inmunizaciones
}

0 Programa Nacional de Imunizações (PNI), criado em 1973 - antes mesmo da criação, em 1988, do Sistema Único de Saúde (SUS) -, foi determinante para o controle bem-sucedido das doenças imunopreveníveis no Brasil. Sua atuação contribuiu sobremaneira para melhorias importantes na situação de saúde da população brasileira. São exemplos: a erradicação da varíola; a eliminação da poliomielite e da febre amarela urbana, da circulação do vírus do sarampo (2016) e da rubéola (2015); assim como a redução da incidência da difteria, da coqueluche, da meningite causada por $H$. influenzae tipo B, do tétano, da tuberculose em menores de 15 anos de idade, e, mais recentemente, das meningites e pneumonias. ${ }^{1}$ A redução da incidência e da mortalidade por doenças imunopreveníveis, especialmente nos primeiros anos de vida, teve notáveis reflexos no aumento da esperança de vida e na redução de hospitalizações. ${ }^{2-4}$

0 rol de vacinas ofertadas pelo SUS foi incrementado ao longo do tempo. Atualmente, são disponibilizadas 19 vacinas para mais de 20 doenças. 0 Calendário Nacional de Vacinação, tal como ocorre nos países desenvolvidos, contempla não apenas as crianças, mas também adolescentes, adultos, idosos, gestantes e povos indígenas. ${ }^{5}$

0 sucesso do PNI e sua crescente complexidade, entretanto, têm se tornado um obstáculo para a manutenção das coberturas vacinais adequadas. À medida que as pessoas não convivem mais com as mortes e incapacidades causadas pelas doenças imunopreveníveis, passam a não mais perceber o risco que estas doenças representam para a sua própria saúde, para os membros de sua família, e para a comunidade. ${ }^{6}$ Nesse cenário, aparecem 0 medo dos eventos adversos e a circulação de notícias falsas sobre os imunobiológicos, que se sobrepõem ao conhecimento sobre a importância e os benefícios das vacinas. Os movimentos antivacina, ${ }^{7}$ embora não sejam muito atuantes no Brasil, estão cada vez mais frequentes e persuasivos, e divulgam informações sem base científica sobre os riscos das vacinas. Ainda, fatores operacionais, como horários restritos de funcionamento das unidades de saúde e o sub-registro das doses aplicadas no Sistema de Informações do Programa Nacional de Imunizações (SI-PNI), dificultam, respectivamente, o acesso aos imunobiológicos e o monitoramento das metas de vacinação.

0 intenso movimento migratório observado em país fronteiriço ao Brasil, inicialmente ocorrido para o estado de Roraima, contribuiu para a propagação do vírus do sarampo, que voltou a circular no país, especialmente nos estados da região Norte. Foram confirmados mais de 10 mil casos da doença em 2018, ${ }^{8}$ e 646 casos, distribuídos em oito estados, até julho de 2019, o que demonstra que esforços adicionais deveriam ser mobilizados para a manutenção de coberturas vacinais adequadas. ${ }^{9}$

A fim de reverter o declínio das coberturas vacinais no Brasil, o Ministério da Saúde lançou o Movimento Vacina Brasil, em 9 de abril de 2019, durante a XXII Marcha a Brasília em Defesa dos Municípios, como parte da agenda dos primeiros 100 dias de governo. A iniciativa tem como objetivo mobilizar os três níveis de gestão e diversos setores da sociedade brasileira e alertar sobre a importância da vacinação como principal medida de controle das doenças imunopreveníveis, e a única capaz de evitar a reemergência de doenças eliminadas no país. Suas primeiras ações foram voltadas para a divulgação da campanha de vacinação contra a influenza, por meio de cartazes, banners, filmes e imagens para uso em mídias sociais. No dia 3 de maio, o Cristo Redentor, no Rio de Janeiro, foi iluminado com a projeção do logotipo do Movimento. Além das ações já realizadas, estão sendo planejadas intensificações de vacinação contra a febre amarela, bem como vacinação contra febre amarela e sarampo nas regiões de fronteira do país, a fim de se ampliarem as coberturas vacinais e o controle das doenças. 
As estratégias para reverter a redução das coberturas vacinais devem considerar os diversos fatores que contribuem para essa situação. A comunicação social, principal estratégia do Movimento Vacina Brasil até o presente, é fundamental para ampliar o acesso às informações baseadas em evidências, especialmente sobre os benefícios da vacinação. Além da divulgação nas mídias tradicionais e eletrônicas, tais estratégias devem contemplar a busca ativa de não vacinados nas populações-alvo, parcerias com escolas e universidades, ampliação dos horários de funcionamento dos postos de vacinação, mobilização da sociedade civil e colaboração das sociedades científicas em parceria com as três instâncias de gestão, bem como o estabelecimento de parcerias intra e intersetoriais. Ademais, fomentar a produção de conhecimento, por meio de inquéritos de coberturas vacinais e de estudos acerca dos fatores associados à não vacinação, considerando-se as diferentes regiões do país, poderá contribuir na definição de estratégias complementares para o enfrentamento da situação atual.

Neste processo, mostram-se como componente fundamental as iniciativas de formação e desenvolvimento em imunizações. Se considerada a existência de mais de 36 mil salas de vacinação no país, tais estratégias devem mobilizar um esforço correspondente para o alcance de milhares de profissionais diretamente envolvidos com os serviços de vacinação, bem como de outros milhões de trabalhadores que, de alguma forma, podem contribuir para 0 alcance das metas estabelecidas. Dessa forma, a implementação de um programa de formação em imunizações depende do empenho das três esferas de gestão do SUS, do necessário engajamento das instituições de ensino públicas e privadas e do apoio dos movimentos sociais e da população em geral, de modo a serem potencializados os efeitos esperados deste esforço.

O protagonismo virtuoso da Rede de Escolas Técnicas, das Escolas de Saúde Pública, dos Centros Formadores vinculados ao SUS e do Ministério da Saúde, em atuação direta ou em parceria com outras instituições, poderá privilegiar o desenvolvimento de estratégias em dois eixos, a seguir especificados.

Eixo 1 - Formação e desenvolvimento de pessoal de nível médio (técnicos e auxiliares de enfermagem, Agentes de Controle de Endemias [ACE] e Agentes Comunitários de Saúde [ACS]), contemplando: (i) produção de materiais de apoio às iniciativas educacionais para a formação destes profissionais (a exemplo de vídeos e cartilhas), com possibilidade de ampla divulgação e múltiplas aplicações; (ii) desenvolvimento de cursos autoinstrucionais, na modalidade de educação a distância $(\mathrm{EAD})$, sobre temas relacionados à vacinação (rede de frio, calendário de vacinação, estratégias para a busca ativa, vacinação de grupos especiais, organização da sala de vacinação, boas práticas em sala de vacinação, SI-PNI); (iii) apoio ao desenvolvimento dos cursos presenciais em parceria com as Secretarias Estaduais e Municipais de Saúde; e (iv) oferta de formação especializada técnica em vacinação para profissionais que atuam em salas de vacina, em parceria com as Secretarias Estaduais e Municipais de Saúde.

Eixo 2 - Formação e desenvolvimento de pessoal de nível superior, incluindo: (i) produção de materiais de apoio às iniciativas educacionais (vídeos, entrevistas, sumários executivos); (ii) indução à produção e disseminação de síntese de evidências científicas que reforcem os benefícios das ações de vacinação e a segurança dos imunobiológicos adotados pelo PNI; (iii) ampla disseminação das publicações técnicas e de suas atualizações (manuais, guias, notas informativas); (iv) desenvolvimento de cursos autoinstrucionais, na modalidade EAD, sobre temas bem estruturados (por exemplo, Calendário Nacional de Vacinação; vacinação de grupos especiais; eventos adversos pós-vacinação; planejamento, monitoramento e avaliação das atividades de vacinação); (v) fortalecimento dos temas relacionados às doenças imunopreveníveis na formação dos profissionais da área da saúde - necessária articulação com a Secretaria da Gestão do Trabalho e da Educação na Saúde do Ministério da Saúde (SGTES/MS) e com o Ministério da Educação (inserção dos temas na graduação; estímulo a iniciativas extracurriculares; articulação com outras iniciativas, como Pro-Residência e o Pro-Internato); (vi) especialização em imunizações, em parceria com a SGTES/MS e Instituições de Ensino Superior; e (vii) estágios avançados em imunizações em instituições nacionais e internacionais de excelência.

As ações da vigilância em saúde, entre as quais se destacam a imunização, são realizadas majoritariamente no âmbito do SUS, de forma descentralizada. Muitas vezes os municípios, particularmente aqueles de menor porte, apresentam dificuldades na execução das ações de vacinação, pela falta de capacitação e pela rotatividade ou escassez dos profissionais que atuam nas salas de vacinação. As estratégias de formação e desenvolvimento deveriam ser também direcionadas aos gestores, inclusive Secretários(as) Municipais de Saúde e prefeitos(as). Quando os gestores têm acesso à informação de qualidade, podem reconhecer o valor das ações de vigilância e lhes dar prioridade, o que repercute positivamente na forma de organização das ações e nas práticas dos profissionais. ${ }^{10}$ 
Reconhecendo a centralidade dos recursos humanos para as ações da vigilância em saúde, em alinhamento com a Política Nacional de Vigilância em Saúde (PNPS) ${ }^{11}$ a formação e o desenvolvimento dos trabalhadores do SUS tornam-se essenciais para 0 alcance das metas de coberturas vacinais. A incorporação dessas estratégias nas próximas etapas do Movimento Vacina Brasil, aliadas às iniciativas já em curso, poderia alcançar milhares de trabalhadores, trabalhadoras, gestores e gestoras do SUS, em todo o território nacional, e impulsionar a reversão do declínio das coberturas vacinais.

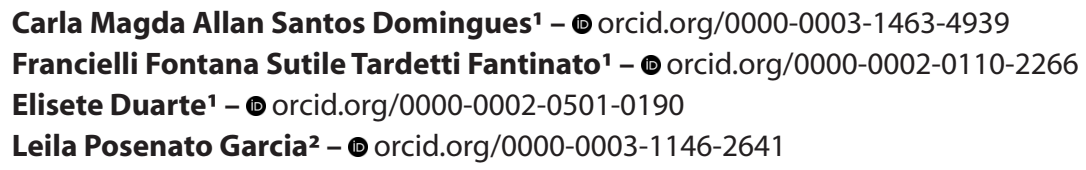

'Ministério da Saúde, Secretaria de Vigilância em Saúde, Brasília, DF, Brasil

2Instituto de Pesquisa Econômica Aplicada, Diretoria de Estudos e Políticas Sociais, Brasília, DF, Brasil

\section{Referências}

1. Ministério da Saúde (BR). Secretaria de Vigilância em Saúde. Departamento de Vigilância das Doenças Transmissíveis. Programa Nacional de Imunizações. Relatório técnico n ${ }^{0}$ 01/2016/CGPNI/DEVIT/SVS/MS: critérios para orientar o processo de decisão para introdução da vacina contra a dengue no Programa Nacional de Imunizações (PNI) [Internet]. Brasília: Ministério da Saúde; 2016 [citado 2019 ago 8]. Disponível em: http://portalarquivos2.saude.gov. br/images/pdf/2016/maio/05/relatorio-01-criterios-orientar-decisao-vacina-dengue.pdf

2. Teixeira AMS, Domingues CMAS. Monitoramento rápido de coberturas vacinais pós-campanhas de vacinação no Brasil: 2008, 2011 e 2012. Epidemiol Serv Saúde [Internet]. 2013 out-dez [citado 2019 ago 8];22(4):565-78. Disponível em: http://scielo.iec.gov.br/pdf/ess/v22n4/v22n4a03.pdf. doi: 10.5123/s1679-49742013000400003

3. Ministério da Saúde (BR). Secretaria de Vigilância em Saúde. Departamento de Vigilância Epidemiológica. Programa Nacional de Imunizações (PNI): 40 anos [Internet]. Brasília: Ministério da Saúde; 2013 [citado 2019 ago 8]. 236 p. Disponível em: http://bvsms.saude.gov.br/bvs/publicacoes/programa_nacional_imunizacoes_pni40.pdf

4. Domingues CMAS, Teixeira AMS. Coberturas vacinais e doenças imunopreveníveis no Brasil no período 1982-2012: avanços e desafios do Programa Nacional de Imunizações. Epidemiol Serv Saúde [Internet]. 2013 jan-mar [citado 2019 ago 8];22(1):9-27. Disponível em: http://scielo.iec.gov.br/pdf/ess/v22n1/v22n1a02.pdf. doi: 10.5123/S167949742013000100002

5. Silva Junior JB. 40 anos do Programa Nacional de Imunizações: uma conquista da Saúde Pública brasileira. Epidemiol Serv Saúde [Internet]. 2013 jan-mar [citado 2019 jul 29];22(1):7-8. Disponível em: http://scielo.iec.gov.br/pdf/ess/ v22n1/v22n1a01.pdf. doi: 10.5123/\$1679-49742013000100001

6. Victora CG. 40 anos do Programa Nacional de Imunizações: o desafio da equidade. Epidemiol Serv Saúde [Internet]. 2013 abr-jun [citado 2019 jul 29];22(2):201-2. Disponível em: http://scielo.iec.gov.br/pdf/ess/v22n2/v22n2a01.pdf. doi: $10.5123 / \$ 1679-49742013000200001$

7. Aps LRMM, Piantola MAF, Pereira SA, Castro JT, Santos FAO, Ferreira LCS. Advents of vaccines and the consequences of non-vaccination: a critical review. Rev Saúde Pública [Internet]. 2018 Apr [cited 2019 Aug 8];52:40. Available from: http://www.scielo.br/pdf/rsp/v52/0034-8910-rsp-S1518-87872018052000384.pdf. doi: 10.11606/s15188787.2018052000384

8. Ministério da Saúde (BR). Secretaria de Vigilância em Saúde. Situação do sarampo no Brasil - 2019.Informe Epidemiol [Internet]. 2019 jan [citado 2019 ago 8];36:1-8. Disponível em: http://portalarquivos2.saude.gov.br/ images/pdf/2019/janeiro/28/Informe-Sarampo-n36-24jan19aed.pdf

9. Ministério da Saúde (BR). Secretaria de Vigilância em Saúde. Sarampo: monitoramento da situação no Brasil - 2019. Informe Epidemiol [Internet]. 2019 jul [citado 2019 ago 8];45:1-6. Disponível em:http://portalarquivos2.saude.gov. br/images/pdf/2019/agosto/01/Informe-Sarampo-n45.pdf 
10. Recktenwaldt M, Junges JR. A organização e a prática da vigilância em saúde em municípios de pequeno porte. Saúde Soc [Internet]. 2017 jun [citado 2019 ago 8];26(2):367-81. Disponível em: http://www.scielo.br/pdf/sausoc/ v26n2/1984-0470-sausoc-26-02-00367.pdf. doi: 10.1590/s0104-12902017171916

11. Brasil. Ministério da Saúde. Conselho Nacional da Saúde. Resolução MS/CNS nº 588, de 12 de julho de 2018. Fica instituída a Política Nacional de Vigilância em Saúde (PNVS) [Internet]. Diário Oficial da União, Brasília (DF), 2018 ago 13 [citado 2019 ago 8];Seção 1:87. Disponível em: http://conselho.saude.gov.br/resolucoes/2018/Reso588.pdf 